\title{
Development of Exon Skipping Therapies for Duchenne Muscular Dystrophy: A Critical Review and a Perspective on the Outstanding Issues
}

\author{
Annemieke Aartsma-Rus,, ${ }^{1,2}$ Volker Straub,2 Robert Hemmings, Manuel Haas,, \\ Gabriele Schlosser-Weber, Violeta Stoyanova-Beninska, Eugenio Mercuri, ${ }^{7}, 8$ \\ Francesco Muntoni, ${ }^{9}$ Bruno Sepodes, ${ }^{10}$ Elizabeth Vroom, ${ }^{11}$ and Pavel Balabanov ${ }^{4}$
}

Duchenne muscular dystrophy (DMD) is a rare, severe, progressive muscle-wasting disease leading to disability and premature death. Patients lack the muscle membrane-stabilizing protein dystrophin. Antisense oligonucleotide (AON)-mediated exon skipping is a therapeutic approach that aims to induce production of partially functional dystrophins. Recently, an AON targeting exon 51 became the first of its class to be approved by the United States regulators [Food and Drug Administration (FDA)] for the treatment of DMD. A unique aspect of the exon-skipping approach for DMD is that, depending on the size and location of the mutation, different exons need to be skipped. This challenge raises a number of questions regarding the development and regulatory approval of those individual compounds. In this study, we present a perspective on those questions, following a European stakeholder meeting involving academics, regulators, and representatives from industry and patient organizations, and in the light of the most recent scientific and regulatory experience.

Keywords: exon skipping, oligonucleotides, regulatory approval

\section{Introduction}

D UCHENNE MUSCULAR DYSTROPHY (DMD) is a rare, severe, progressive muscle-wasting disease. Continuous efforts to improve the understanding of the disease and to investigate potential approaches to treat it have brought the field to the stage of clinical trials and marketing authorization applications. The most advanced therapies include those that aim at restoration of the missing protein, dystrophin, using mutation-specific genetic approaches, such as antisense oligo- nucleotide (AON)-mediated exon skipping (see Box 1: Duchenne Muscular Dystrophy and Exon Skipping). These developments revealed some as of yet unresolved issues, such as the identification and use of biomarkers in DMD, the fact that each AON applies to only a subgroup of patients with this condition, and the best way to fulfill the criteria for regulatory approval for the different AONs. The current perspective on these and other issues was discussed in detail during a DMD multistakeholder meeting involving representatives from patient organizations, academia, industry, and European Union

\footnotetext{
${ }^{1}$ Department of Human Genetics, Leiden University Medical Center, Leiden, the Netherlands.

${ }^{2}$ John Walton Muscular Dystrophy Research Centre, Institute of Genetic Medicine, Newcastle University, Newcastle upon Tyne, United Kingdom.

${ }_{3}^{3}$ Medicines and Healthcare Product Regulatory Agency, London, United Kingdom.

${ }^{4}$ Central Nervous System and Ophthalmology, Scientific and Regulatory Management Department, Human Medicines Evaluation Division, European Medicines Agency, London, United Kingdom.

${ }_{5}^{5}$ Bundesinstitut für Arzneimittel und Medizinprodukte, Bonn, Germany.

${ }^{6}$ Medicines Evaluation Board, Utrecht, the Netherlands.

${ }^{7}$ Department of Pediatric Neurology, Catholic University, Rome, Italy.

${ }^{8}$ Centro Clinico Nemo, Policlinico Gemelli, Rome, Italy.

${ }^{9}$ Dubowitz Neuromuscular Center, UCL Great Ormond Street Institute of Child Health, London, United Kingdom.

${ }^{10}$ Faculdade de Farmácia, Universidade de Lisboa, Lisboa, Portugal.

${ }^{11}$ United Parent Project Muscular Dystrophy, Amsterdam, the Netherlands.

(c) Annemieke Aartsma-Rus et al. 2017; Published by Mary Ann Liebert, Inc. This is an Open Access article distributed under the terms of the Creative Commons Attribution License, which permits unrestricted use, distribution, and reproduction in any medium, provided the original work is properly cited.
} 
Box 1: Duchenne Muscular Dystrophy and Exon Skipping

DMD is an X-linked, progressive muscle wasting disease that affects 1 in 5,000 newborn males [2]. The disease is caused by mutations in the $D M D$ gene that abolish the production of dystrophin protein [3]. Dystrophin links the F-actin in the cytoskeleton with beta-dystroglycan and the extracellular matrix, through its $\mathrm{N}$ - and C-terminal domains, respectively [4]. Mutations causing the premature truncation of dystrophin translation result in nonfunctional and unstable dystrophin proteins typically undetectable with standard diagnostic techniques. However, internal deletions or duplications maintaining the reading frame allow the production of dystrophins that maintain their $\mathrm{N}$ - and $\mathrm{C}$-terminal domains. These dystrophins are more stable and partially functional and are associated with a less severe and less progressive allelic form of muscular dystrophy, Becker muscular dystrophy (BMD) [5].

Over 7,000 different mutations have been reported for DMD patients [6,7]. Most patients $(\sim 65 \%)$ carry large deletions involving one or more exons, but large duplications $(\sim 12 \%)$ and small mutations $(20 \%)$ are frequently reported as well. The commonality of these mutations is that they all result in nonfunctional dystrophins. However, many DMD patients can unexpectedly produce trace amounts of BMD-type dystrophins [8].

For example, a deletion of exon 45 is one of the most common deletions found in DMD patients, whereas a deletion of exons 44 and 45 is generally associated with BMD [9]. Thus, if exon 44 could be bypassed in pre-messenger RNA (mRNA) transcripts of these DMD patients, this would restore the reading frame and enable the production of a partially functional BMD-like dystrophin (Fig. 1). In fact, it appears that many patients with a deletion bordering on exon 44, skip exon 44 spontaneously, although at very low levels. This results in slightly increased levels of dystrophin when compared with DMD patients carrying other deletions, and most likely underlies the less severe disease progression observed in these patients compared with DMD patients with other deletions [9-11].

The rationale of antisense-mediated exon skipping is to induce the skipping of a target exon to restore the reading frame (Fig. 1) [12]. For patients with an exon 45 deletion, this would require an AON targeting exon 44 . However, different patients have different mutations and exon 44 skipping would only apply to $\sim 8 \%$ of patients [7,12]. To develop the exon-skipping approach for larger groups of patients, AONs targeting additional exons of the gene would have to be developed, for example, AONs to skip exon 51, which applies to $13 \%-14 \%$ of patients, that is, the largest group of patients $[7,12]$.

About $20 \%$ of DMD patients have deletions that remove critical domains of the dystrophin protein. For these restoring the reading frame is anticipated not to be therapeutic, since the resulting dystrophin will not be functional, and that patients with in-frame mutations deleting these domains have DMD rather than BMD [6,8]. Theoretically, AONs could also be used to induce exon skipping of in-frame exons containing nonsense mutations; indeed this is the strategy commonly used in the experimental studies in the dystrophic $m d x$ mouse, carrying a nonsense mutation in the in-frame exon 23 . For a more in-depth discussion of the applicability of exon skipping for different mutation types, we refer the reader to a recent review [8].

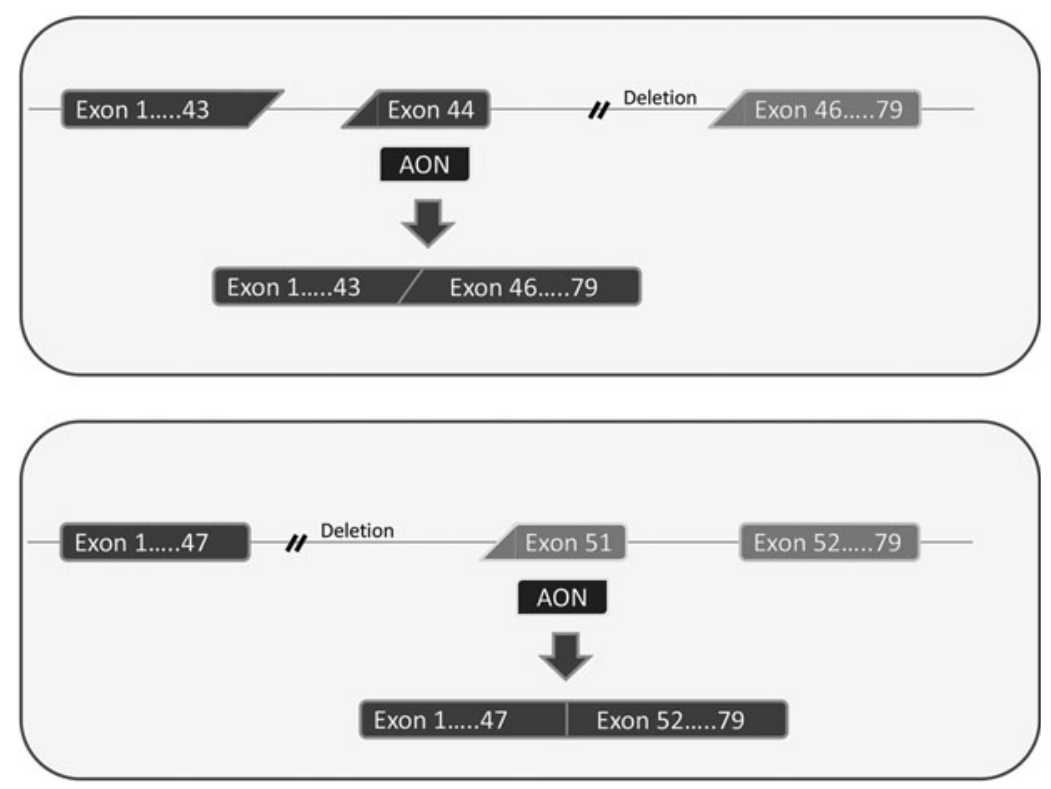

FIG. 1. Schematic depiction of the reading frame rule and the exon-skipping approach. Top panel: a deletion of exon 45 disrupts the reading frame (exon 44 and 46 do not "fit") and is associated with DMD, whereas a deletion of exon 44 and 45 maintains the reading frame (exon 43 and 46 would "fit") and is associated with BMD. Using AONs, the pre-mRNA splicing process can be manipulated. In this example AONs targeting exon 44 hide this exon from the splicing machinery, causing exon 43 to be joined to exon 46, which restores the reading frame and allows production of a BMD-like dystrophin. Bottom panel: antisense-mediated exon skipping is a mutation-specific approach. For example, a deletion of exon 48-50 requires the skipping of exon 51 to restore the reading frame and allow the production of a BMD-like dystrophy. AONs, antisense oligonucleotide; BMD, Becker muscular dystrophy; DMD, Duchenne muscular dystrophy; mRNA, messenger RNA. 
(EU) drug regulatory agencies. A policy view, highlighting the main learnings for orphan drug development in EU, and stressing the importance of stakeholder communication, has been published as a result of this collaboration [1].

In this perspective it is our intention to continue and outline the discussion pertaining specifically to the exon-skipping approach in DMD, discuss the relevant regulatory topics and critical questions, such as the use of adequate biomarkers as potential surrogate markers in AON drug development, and whether class approval is possible for DMD AONs. We will also include our recommendations on how to best approach the centralized regulatory system in the EU to minimize time and resources for drug developers, and guarantee that drugs, benefiting patients suffering from this devastating condition, reach them in the fastest way possible.

\section{From Proof-of-Concept to Drug Approval: Current Experience}

Proof-of-concept that the antisense exon-skipping approach indeed could restore dystrophin protein expression came from tests in patient and animal model-derived cell culture experiments and local AON injections in dystrophic mice [13-15], followed by systemic treatment experiments in mouse and dog models [16-18]. AONs have been developed to skip each dystrophin exon $[19,20]$. However, since exon 51 AONs apply to the largest subgroup of patients, they were the first to be transferred into clinical programs. Two AONs, based on different chemistries, were evaluated in various clinical trials, a $2^{\prime} O$-methyl phosphorothioate (2OMePS)modified AON-drisapersen and a phosphorodiamidate morpholino oligomer (PMO) - eteplirsen [21-30]. Both compounds were able to restore dystrophin expression after local injection [26,29]. In the clinical setting each compound followed a different clinical program.

Drisapersen has been tested in various clinical trials in over 300 patients for up to 4 years, most often using a $6 \mathrm{mg} / \mathrm{kg}$ weekly subcutaneous injection regimen [21,24-26,30]. In two small placebo-controlled studies, younger patients (6-8 years) treated with drisapersen walked a longer distance in 6 min (six-minute walk distance [6MWD]) as compared with placebo, although this difference was not statistically significant and not observed in a large phase 3 trial involving a more heterogeneous patient population, for example, patients aged 5-16 years. Side effects included injection site reactions (severe in some cases), severe thrombocytopenia in a small percentage of treated subjects, and significant renal effects in another small subset of treated subjects.

Marketing authorization applications were filed with the Food and Drug Administration (FDA) and the European Medicines Agency (EMA) mainly based on post hoc analysis in selected cohorts, where a therapeutic effect was speculated to be most likely measurable by the 6MWD. The FDA declared that drisapersen was not approvable on the basis of data presented in the new drug application, whereas the application at EMA was withdrawn by the applicant before the evaluation was completed [31,32]. At the same time, it was announced that the clinical development of drisapersen was stopped, in lieu of the identification of the next generation of exon 51 skipping AONs [21,31].

Eteplirsen, another AON targeting exon 51-skippable DMD mutations in patients, has been tested in multiple clinical trials, generally using a dosing regimen of weekly intravenous doses of 30 or $50 \mathrm{mg} / \mathrm{kg}$ [21,27,28]. In an open-label study that has been ongoing for over 4 years, 10 ambulant US patients appeared to have a slower disease progression than matched untreated natural history controls from Italy and Belgium as measured by the 6MWD [28]. No clear treatment-related side effects were observed.

Eteplirsen recently received accelerated approval from the FDA, based on pharmacodynamic effects with minimal increases in dystrophin levels in biopsies from some treated patients, with binding postapproval commitments issued by the FDA to assess functional effects and clinical benefit in additional trials before 2021 [21,33]. This application was more recently submitted to EMA and is currently under review.

While eteplirsen approval provides a treatment option for $\sim 13 \%$ of DMD patients in the United States, still no AONs are available for the majority of patients, and AONs targeting additional exons are still in the development phase. To increase the availability of exon skipping medicinal products in clinical practice that could potentially address the needs of larger groups of DMD patients, more data need to be gathered from all aspects of pharmaceutical development for these AONs.

\section{Clinical Development of Additional AONs Beyond the First Approved AON: Is There Room for Extrapolation?}

Media have widely covered the controversy related to the FDA approval of the first AON in DMD (eteplirsen) [21]. Nevertheless, the approval brought hope that the AON approach is indeed viable for this condition and should be a stimulus for further research and development in the AON field. The clinical development of drisapersen has shown that placebo-controlled trials $[21,30]$ are feasible for some of the subgroups of DMD patients. Currently, exon-skipping trials focus on exons that could be skipped in 6\%-14\% of patients for each product [12], where the numbers are sufficient to conduct trials in internationally standardized cohorts.

Due to the variability of clinical course in DMD individuals, the already relatively advanced stage of disease when AONs are started in clinical trials, relatively large cohorts of affected individuals are required to unequivocally demonstrate clinical efficacy. These developments are expected to provide a significant amount of data on the clinical behavior of various AONs in DMD that could then be used to properly support the development of a number of additional AONs that apply to smaller patient numbers.

When dealing with trials that address smaller patient populations, the clinical development becomes more challenging, because there will be fewer eligible patients, with less opportunity to recruit a homogeneous patient population, for example, with respect to age and disease stage. This will probably hamper the performance of randomized placebo-controlled trials, not only because of eligibility and recruitment issues, and the expected length of the trials, but also because of problems related to the interpretation of the observed treatment effects.

In cases where AONs have already been approved, and where comprehensive data on their efficacy and safety are available, the requirements to approve additional AONs of the same chemistry may change, based on the accumulated evidence and knowledge about the licensed medicinal 
products. In these cases, partial extrapolation might be a possible strategy, to supplement the available data for a specific AON in development, with data that are likely to be applicable to all members of the class, for example, certain common safety characteristics. The consideration of such an approach would, however, rely heavily on having a clear understanding of the disease progression in the specific subgroup of patients (depending on their characteristics, eg, genetic defect), and of the pharmacology of the compound and its expected effect size in both pharmacodynamic markers and in clinical endpoints.

Despite the fact that it is expected that the AONs of a given chemistry should have comparable pharmacokinetic and pharmacodynamic properties, these will not be necessarily identical. The level of difference will vary according to chemical modifications, and sequence composition of the AONs, which may result in a significant difference in the AONs' clinical behavior.

Nevertheless, certain characteristics of the AONs' chemistry could give experts grounds to believe that the different compounds will share a number of common properties. The PMO chemistry is charge neutral and so far only very limited differences have been observed between AONs of different lengths and/or sequence composition in pharmacokinetic and pharmacodynamic properties in preclinical safety tests [34].

The 2OMePS-modified AONs are negatively charged and can bind serum proteins with varying affinity based on AON length and/or nucleotide composition. Hence, while the overall class characteristics for phosphorothioate oligonucleotides are similar, there can be quantitative differences, for example, in biodistribution and side effect profiles, which have been observed in animal models and clinical trials [35]. Furthermore, certain sequence motifs $(\mathrm{CpG})$ have been reported to be associated with inflammation [36], although these can be avoided with specific AON designs.

Finally, while generally there is good correlation between activity in cultured cells and animal models for AONs, exceptions have been reported [18]. For all of the abovementioned reasons, it is expected that a certain amount of preclinical and clinical data will always have to be generated to confirm the hypothesized efficacy and make assumptions on the expected safety profile.

The discussion on the appropriateness of potential extrapolation of data from other AONs will have to take into account the totality of data generated during the whole drug development. Ensuring the availability of as much robust data as possible, to clearly establish the safety pharmacology, pharmacokinetic behavior, and pharmacodynamic effects of the drug, will be necessary to help identify the gaps that could benefit from any type of extrapolation. The level and appropriateness of potential extrapolation may be discussed in advance. In fact, it is strongly recommended that the intended drug development, trial designs, and extrapolation strategies be discussed at an early stage with the regulators through the available platforms for scientific dialogue [37].

In addition to developing AONs for additional exons, developing more efficient AONs for the same exons is another possible drug development strategy. It has to be kept in mind that the orphan drug regulation stipulates that a company with successful marketing authorization of their medicinal product acquires EU market exclusivity for 10 years [38].
In practice, this poses several challenges for future developments. For instance any new AON targeting the same exon (eg, of a different chemistry but aiming at the same target population) may either be concluded to be similar or nonsimilar in the context of the orphan legislation. When it is similar, the AON may come to the market through a derogation, in which case significant benefit would still need to be established. When the AON would be concluded not to be similar, it can come to the market as an orphan medicinal product if providing significant benefit for the patients, as defined in the legislation [39]. The provision of significant benefit is not limited to improved clinical efficacy, but could also mean an improved safety profile, or a more tolerable route of administration.

\section{Development of AONs for Very Small Groups of Patients}

Both drisapersen and eteplirsen, (which induce exon 51 skipping), apply to only $13 \%-14 \%$ of DMD patients. To allow for treatment of additional groups of patients, additional AONs targeting other exons have to be developed and marketed. Currently, clinical trials with AONs targeting exon 45 and 53 have been initiated [40], because a separate development program has to be performed for each compound. For exons where skipping applies to relatively larger patient groups, this is not an insurmountable problem. However, for most exons, the applicability per exon is $<1 \%$ of patients (eg, exon 49 skipping would apply to only $0.02 \%$ of all patients), which poses challenges in their clinical development [41].

While large, controlled clinical studies may not be feasible for the limited patient numbers of certain DMD subpopulations, drug developers should focus on collecting as much information as possible that will allow for a benefit/risk evaluation to be performed by the decision makers on the specific AON (both in terms of initial regulatory approval and later on for reimbursement discussions). It is recommended that in cases where data are impossible to collect, methods of extrapolation based on data-driven scientific methods should be considered.

The DMD community has put forward the proposal for "platform approval" or "class approval" as a way to tackle the challenge of developing AONs for small groups of patients. In short, this represents the idea to seek approval for all AONs of certain chemistry as a group and not as individual applications. The DMD community expected that this could be discussed with the regulatory agencies after sufficient safety and efficacy data had been obtained for a number of DMD exon-skipping AONs. However, according to the current legislation in the EU, different medicinal products are evaluated and authorized individually, on the basis of their specific risks and benefits (article 6 and 8 of the Directive 2001/83/EC of the legislation [42]).

The assessment of what constitutes a separate medicinal product is not limited only to the differences in the active substance, but also considers factors, such as production process, pharmaceutical form, excipients, and others, and even legal provisions such as ownership of the rights to market the product. From a regulatory and scientific perspective, the fact that the different AONs target different exons, would likely qualify them as different medicinal products. 
Even in the case where the same exon is targeted, it could be argued that AONs of similar chemistry (ie, belonging to the same class) may act in a different manner based on differences in their composition and length, which may result in differences in their pharmacokinetic and pharmacodynamic profiles, and as a consequence may also have different efficacy and safety outcomes. This will also qualify them as different medicinal products that will need to take the regulatory route to approval separately. Nevertheless, some level of extrapolation based on accumulated data from previously developed AONs could be discussed. It must always be kept in mind, however, that these data will have to be legally available to the applicant to be used in different regulatory submissions (marketing application dossiers).

\section{Dystrophin Restoration as a Biomarker for Small Trials}

Exon skipping aims to restore or increase dystrophin expression in DMD patients' muscle fibers. Dystrophin quantification was therefore considered as an obvious pharmacodynamic biomarker, but to be used in clinical development as a surrogate for functional outcome, ideally this biomarker needs to be validated. This means that evidence for the correlation between dystrophin levels and clinical outcomes is required. Validating dystrophin quantification is not a straightforward process $[43,44]$, and involves dealing with a number of specific issues:

(1) Dystrophin is a large protein that is expressed at very low levels in skeletal muscles, which makes quantification difficult, also because there is no reference standard that can be utilized.

(2) Dystrophin levels vary among unaffected individuals, but also among muscles and muscle fibers within a certain individual.

(3) The progressive replacement of muscle by fibrotic and adipose tissue in DMD patients hampers absolute quantification, since these tissues do not express dystrophin. Moreover there is variability of muscle pathology between muscles, but also within individual muscles.

(4) A muscle biopsy involves a tiny sample of a single muscle. First, it is possible that a biopsy is obtained only containing very few muscle fibers, which makes quantification impossible. Second, determination of the pattern and level of dystrophin expression in a particular muscle biopsy of a given muscle in a given patient may not necessarily be representative of every single muscle in the same patient.

(5) Different internally shortened dystrophins will be formed through exon skipping for patients with different deletions (eg, the dystrophin formed after skipping exon 51 will differ for a deletion of exon 49-50 vs. a deletion of exon 52 and both will differ from the dystrophin formed after exon skipping for a deletion of exon 45). For most of these dystrophin molecules it is known that they may be functional because they are found in muscles of BMD individuals [6]. However, the functionality of other rare in-frame mutations has never been proven as the corresponding BMD patients remain uncharacterized. In such cases, it will be important to confirm the restoration of the proper localization of dystrophin-associated proteins in a muscle biopsy by immunofluorescence analysis.

(6) While it is known that the presence of low levels of dystrophin since birth are associated with a slower disease progression [10,11], dystrophin expression induced by exon skipping could only become effective at the time of intervention, when the pathological process in the muscles have already led to substantial damage and loss of muscle mass leading to muscle weakness. A newly produced truncated form of dystrophin needs to be correctly localized and integrated in the protein complex to become functional. It is not known whether this occurs in the context of a dystrophic muscle in a DMD patient in the same way as in a BMD patient. As it is anticipated that the muscle quality at the time of intervention will influence the therapeutic effect, it cannot be assumed that restoring the similar low levels of dystrophin will cause the same effect on disease progression as having low levels of dystrophin from birth.

Currently, the available data are insufficient to establish a correlation between dystrophin levels and clinical function for various stages of disease. The only indirect inference derives from different animal models in which dystrophin restoration was induced at different stages of muscle pathology [45]. While these studies have demonstrated functional benefit for dystrophin re-expression, it is not clear how to translate these preclinical data to the human scenario.

In the regulatory submissions up to now, eteplirsen was approved in the United States based on an increase in dystrophin expression in eteplirsen-treated patients, and even if the levels of expression were very low [21] dystrophin expression served as a surrogate endpoint for this approval.

The current EMA regulatory position on the subject, as reflected in the published EMA DMD guideline [46], is that the quantification of dystrophin expression still has limitations that prevent its use as a surrogate measure for efficacy, that is, to be used as the only endpoint in a confirmatory trial.

This is due to two main reasons: the first is related to the limitations of dystrophin quantification in muscle biopsies (as discussed earlier in this section); the second is related to the fact that a clear relationship between the restoration of low levels of dystrophin and a functional outcome, clinically most important to the patients, has not been established so far. As such, the levels of dystrophin are not considered sufficiently validated in the regulatory sense, for the context of use as primary efficacy endpoints. However, in cases where the mechanism of action of the therapy is related to the restoration of dystrophin expression, detection of dystrophin in muscle tissue could serve as a pharmacodynamic marker for proof of concept.

Drug developers should be aware that a dedicated procedure is in place at EMA for the qualification of biomarkers for a specific intended use in the context of research and development into pharmaceuticals [47]. This procedure provides a platform for discussion on any potential novel methodology that is to be used in the drug development process. During a continuous early dialogue, the gaps that need to be filled by appropriate data will be identified, and the appropriate context of use determined. The specific biomarker/ 
tool is then officially validated/qualified for its use in regulatory submissions.

\section{Regulatory Tools and Incentives in the Centralized EU Regulatory System}

Examples from other fields show that early engagement with the regulatory authorities has proven to be beneficial in providing useful guidance and scientific advice, leading to a higher success rate of subsequent marketing authorizations $[48,49]$. It is useful to be aware of the platforms for early dialogue, and of the respective incentives that the EMA provides, to better guide drug development and guarantee less queries during potential future assessment. Here, the main features of the platforms that may be most relevant for AON developers are introduced:

\section{Innovation Task Force}

The Innovation Task Force (ITF) [50] is a multidisciplinary group that includes scientific, regulatory, and legal competences. It is a discussion platform for early dialogue with applicants, in particular micro-, small-, and mediumsized enterprises (SMEs) to proactively identify scientific, legal, and regulatory issues of emerging therapies and technologies. This format also allows for a face-to-face meeting with relevant experts.

\section{Priority medicines scheme}

Priority medicines (PRIME) [51] is a scheme launched by EMA to enhance support for the development of medicines that target an unmet medical need. This voluntary scheme is based on enhanced interaction and early dialogue with developers of promising medicines, to optimize development plans and speed up evaluation so these medicines can reach patients earlier.

\section{Scientific advice and protocol assistance}

EMA also provides scientific advice and protocol assistance to companies [37]. EMA can give scientific advice to a company on the appropriate tests and studies in the development of a medicine. This is designed to facilitate the development and availability of high-quality, effective, and acceptably safe medicines, for the benefit of patients. Companies can request scientific advice from the EMA at any stage of development of a medicine, regardless of whether the medicine is eligible for the centralized authorization procedure.

Scientific advice helps the company to make sure that it performs the appropriate tests and studies, so that no major objections regarding the design of the tests are likely to be raised during evaluation of the marketing authorization application. Companies developing orphan medicines for rare diseases can receive answers to questions relating to the criteria for authorization of an orphan medicine, and once they have orphan drug designation also have access to protocol assistance [52].

\section{Orphan designation}

Rare diseases are defined as life-threatening or chronically debilitating conditions that affect no more than 5 in
10,000 people in the EU, equivalent to fewer than 250,000 people for each disease. Medicines developed for rare diseases are termed "orphan medicines" in the regulatory world. To support drug development in these conditions, the EU offers a range of incentives to encourage the development of these medicines [52]. In addition to the main regulatory incentive of 10-year market exclusivity already mentioned before, these also include significant fee reductions, as well as administrative and regulatory support, especially for SMEs [53].

\section{Small- and medium-sized enterprises}

EMA addresses the unique needs of SMEs through the SME office [54]. This dedicated interface has the sole remit of providing regulatory, financial, and administrative assistance to small pharmaceutical companies. This includes a wide range of activities to support SMEs, such as direct assistance on regulatory matters, fee reductions and exemptions, assistance with translations, inclusion in an online SME register, and workshops and training sessions.

\section{Future Perspective}

The therapeutic development of AON-mediated exon skipping for DMD has come a long way. Exon 51 skipping is now an approved treatment in the United States, while evaluation by EMA is pending. However, this applies only to $13 \%$ of DMD patients and thus far only those in the United States. Developing additional AONs targeting other exons will be needed to increase the number of patients to which this approach applies. For AONs applying to smaller groups of patients, this will be increasingly challenging. Some proposals, such as platform approval, sound on face value appealing from a scientific perspective, but do not comply with the current regulatory system and legislation, whereby different medicinal products are individually assessed.

Nevertheless, as knowledge obtained for AONs in DMD continues to accumulate, extrapolation approaches may be discussed with regulatory authorities to try and accelerate the development of future AONs. These therapies not only represent a potentially disease-modifying path for one of the most devastating neuromuscular diseases, but also help us to foresee a future for personalized genetic therapies in neurology [55].

\section{Acknowledgments}

This report was written based on a stakeholder workshop involving academics, industry, patient representatives, and regulators, which was sponsored by COST Action BM1207 (www.exonskipping.eu) and SCOPEDMD (www.scope-dmd.eu), and hosted by the European Medicines Agencies.

\section{Author Disclosure Statement}

The views expressed in this article are the personal views of the authors and may not be understood or quoted as being made on behalf of or reflecting the position of the agencies or organizations with which the authors are affiliated. A.A.R. discloses being employed by LUMC, which has patents on exon-skipping technology. As coinventor of some of these patents A.A.R. is entitled to a share of royalties. A.A.R. 
further discloses being ad hoc consultant for PTC Therapeutics, BioMarin Pharmaceuticals, Inc., Guidepoint Global and GLG consultancy, Grunenthal, Wave Therapeutics and BioClinica, having been a member of the Duchenne Network Steering Committee (BioMarin Pharmaceuticals, Inc.) and being a member of the scientific advisory boards of ProQR and Philae Pharmaceuticals. Remuneration for these activities is paid to LUMC. LUMC also received speaker honoraria from PTC Therapeutics and BioMarin Pharmaceuticals, Inc. V.S. is or has been a principal investigator for trials sponsored by Biogen, GSK, Ionis Pharmaceuticals, Prosensa/BioMarin Pharmaceuticals, Inc., Sanofi Genzyme and Sarepta Therapeutics. He has received speaker honoraria from Sanofi Genzyme and is or has over the last 3 years been on advisory boards for Audentes Therapeutics, Biogen, BioMarin Pharmaceuticals, Inc., Bristol-Myer Squibb, Italfarmaco SpA, Sarepta Therapeutics, Summit Therapeutics, Tivorsan, TrophyNOD, and Wave Therapeutics. He has research collaborations with Ultragenyx and Sanofi Genzyme. F.M. is supported by the National Institute of Health Research Biomedical Research Center at Great Ormond Street Hospital for Children NHS Foundation Trust. He is or has been a principal investigator for trials sponsored by Biogen; GSK; Ionis Pharmaceuticals; Prosensa/BioMarin Pharmaceuticals, Inc.; Pfizer; PTC Therapeutics; Roche; Sarepta Therapeutics; Audentes; EspeRare; Summit Therapeutics; and Genethon. Over the last 3 years he has been on advisory boards for Pfizer; Akashi Therapeutics, Biogen; Catabasis, Italfarmaco, Roche, Sarepta Therapeutics, Tivorsan, Wave Therapeutics, and Avexis. All other authors declare no conflicts of interest.

\section{References}

1. Straub V, P Balabanov, K Bushby, M Ensini, N Goemans, LA De, A Pereda, R Hemmings, G Campion, et al. (2016). Stakeholder cooperation to overcome challenges in orphan medicine development: the example of Duchenne muscular dystrophy. Lancet Neurol 15:882-890.

2. Moat SJ, DM Bradley, R Salmon, A Clarke and L Hartley. (2013). Newborn bloodspot screening for Duchenne muscular dystrophy: 21 years experience in Wales (UK). Eur J Hum Genet 21:1049-1053.

3. Hoffman EP, RH Brown, Jr and LM Kunkel. (1987). Dystrophin: the protein product of the Duchenne muscular dystrophy locus. Cell 51:919-928.

4. Koenig M, AP Monaco and LM Kunkel. (1988). The complete sequence of dystrophin predicts a rod-shaped cytoskeletal protein. Cell 53:219-228.

5. Monaco AP, CJ Bertelson, S Liechti-Gallati, H Moser and LM Kunkel. (1988). An explanation for the phenotypic differences between patients bearing partial deletions of the DMD locus. Genomics 2:90-95.

6. Aartsma-Rus A, JC Van Deutekom, IF Fokkema, GJ Van Ommen and JT Den Dunnen. (2006). Entries in the Leiden Duchenne muscular dystrophy mutation database: an overview of mutation types and paradoxical cases that confirm the reading-frame rule. Muscle Nerve 34:135-144.

7. Bladen CL, D Salgado, S Monges, ME Foncuberta, K Kekou, K Kosma, H Dawkins, et al. (2015). The TREATNMD DMD Global Database: analysis of more than 7,000 Duchenne muscular dystrophy mutations. Hum Mutat 36: 395-402.
8. Niks EH and A Aartsma-Rus. (2017). Exon skipping: a first in class strategy for Duchenne muscular dystrophy. Expert Opin Biol Ther 17:225-236.

9. Anthony K, V Arechavala-Gomeza, V Ricotti, S Torelli, L Feng, N Janghra, G Tasca, M Guglieri, R Barresi, et al. (2014). Biochemical characterization of patients with inframe or out-of-frame DMD deletions pertinent to exon 44 or 45 skipping. JAMA Neurol 71:32-40.

10. Pane M, ES Mazzone, MP Sormani, S Messina, GL Vita, L Fanelli, A Berardinelli, et al. (2014). 6 Minute walk test in Duchenne MD patients with different mutations: 12 month changes. PLoS One 9:e83400.

11. van den Bergen JC, HB Ginjaar, EH Niks, A Aartsma-Rus and JJ Verschuuren. (2014). Prolonged ambulation in Duchenne patients with a mutation amenable to exon 44 skipping. J Neuromuscul Dis 1:91-94.

12. Aartsma-Rus A, I Fokkema, J Verschuuren, I Ginjaar, DJ van, GJ Van Ommen and JT Den Dunnen. (2009). Theoretic applicability of antisense-mediated exon skipping for Duchenne muscular dystrophy mutations. Hum Mutat 30: 293-299.

13. Mann CJ, K Honeyman, AJ Cheng, T Ly, F Lloyd, S Fletcher, JE Morgan, TA Partridge and SD Wilton. (2001). Antisense-induced exon skipping and synthesis of dystrophin in the mdx mouse. Proc Natl Acad Sci U S A 98: 42-47.

14. McClorey G, HM Moulton, PL Iversen, S Fletcher and SD Wilton. (2006). Antisense oligonucleotide-induced exon skipping restores dystrophin expression in vitro in a canine model of DMD. Gene Ther 13:1373-1381.

15. Van Deutekom JC, M Bremmer-Bout, AA Janson, IB Ginjaar, F Baas, JT Den Dunnen and GJ Van Ommen. (2001). Antisense-induced exon skipping restores dystrophin expression in DMD patient derived muscle cells. Hum Mol Genet 10:1547-1554.

16. Aoki Y, A Nakamura, T Yokota, T Saito, H Okazawa, T Nagata and S Takeda. (2010). In-frame dystrophin following exon 51-skipping improves muscle pathology and function in the exon 52-deficient mdx mouse. Mol Ther 18:1995-2005.

17. Lu QL, A Rabinowitz, YC Chen, T Yokota, H Yin, J Alter, A Jadoon, G Bou-Gharios and T Partridge. (2005). Systemic delivery of antisense oligoribonucleotide restores dystrophin expression in body-wide skeletal muscles. Proc Natl Acad Sci U S A 102:198-203.

18. Yokota T, QL Lu, T Partridge, M Kobayashi, A Nakamura, S Takeda and E Hoffman. (2009). Efficacy of systemic morpholino exon-skipping in Duchenne dystrophy dogs. Ann Neurol 65:667-676.

19. Aartsma-Rus A, CL De Winter, AA Janson, WE Kaman, GJ Van Ommen, JT Den Dunnen and JC Van Deutekom. (2005). Functional analysis of 114 exon-internal AONs for targeted DMD exon skipping: indication for steric hindrance of SR protein binding sites. Oligonucleotides 15: 284-297.

20. Wilton SD, AM Fall, PL Harding, G McClorey, C Coleman and S Fletcher. (2007). Antisense oligonucleotide-induced exon skipping across the human dystrophin gene transcript. Mol Ther 15:1288-1296.

21. Aartsma-Rus A and AM Krieg. (2017). FDA approves eteplirsen for Duchenne muscular dystrophy: the next chapter in the eteplirsen saga. Nucleic Acid Ther 27:1-3.

22. Cirak S, V Arechavala-Gomeza, M Guglieri, L Feng, S Torelli, K Anthony, S Abbs, ME Garralda, J Bourke, et al. 
(2011). Exon skipping and dystrophin restoration in patients with Duchenne muscular dystrophy after systemic phosphorodiamidate morpholino oligomer treatment: an open-label, phase 2, dose-escalation study. Lancet 378: 595-605.

23. Cirak S, L Feng, K Anthony, V Arechavala-Gomeza, S Torelli, C Sewry, JE Morgan and F Muntoni. (2012). Restoration of the dystrophin-associated glycoprotein complex after exon skipping therapy in Duchenne muscular dystrophy. Mol Ther 20:462-467.

24. Goemans NM, M Tulinius, JT van den Akker, BE Burm, PF Ekhart, N Heuvelmans, T Holling, AA Janson, GJ Platenburg, et al. (2011). Systemic administration of PRO051 in Duchenne's muscular dystrophy. N Engl J Med 364: 1513-1522.

25. Goemans NM, M Tulinius, $M$ van den Hauwe, AK Kroksmark, G Buyse, RJ Wilson, JC Van Deutekom, SJ de Kimpe, A Lourbakos and G Campion. (2016). Long-term efficacy, safety, and pharmacokinetics of drisapersen in Duchenne muscular dystrophy: results from an open-label extension study. PLoS One 11:e0161955.

26. Kinali M, V Arechavala-Gomeza, L Feng, S Cirak, D Hunt, C Adkin, M Guglieri, E Ashton, S Abbs, et al. (2009). Local restoration of dystrophin expression with the morpholino oligomer AVI-4658 in Duchenne muscular dystrophy: a single-blind, placebo-controlled, doseescalation, proof-of-concept study. Lancet Neurol 8: 918-928.

27. Mendell JR, LR Rodino-Klapac, Z Sahenk, K Roush, L Bird, LP Lowes, L Alfano, AM Gomez, S Lewis, et al. (2013). Eteplirsen for the treatment of Duchenne muscular dystrophy. Ann Neurol 74:637-647.

28. Mendell JR, N Goemans, LP Lowes, LN Alfano, K Berry, J Shao, EM Kaye and E Mercuri. (2016). Longitudinal effect of eteplirsen versus historical control on ambulation in Duchenne muscular dystrophy. Ann Neurol 79: 257-271.

29. Van Deutekom JC, AA Janson, IB Ginjaar, WS Frankhuizen, A Aartsma-Rus, M Bremmer-Bout, JT Den Dunnen, K Koop, AJ van der Kooi, et al. (2007). Local dystrophin restoration with antisense oligonucleotide PRO051. N Engl J Med 357:2677-2686.

30. Voit T, H Topaloglu, V Straub, F Muntoni, N Deconinck, G Campion, SJ de Kimpe, M Eagle, M Guglieri, et al. (2014). Safety and efficacy of drisapersen for the treatment of Duchenne muscular dystrophy (DEMAND II): an exploratory, randomised, placebo-controlled phase 2 study. Lancet Neurol 13:987-996.

31. Press release BioMarin on withdrawal drisapersen. http:// investors.biomarin.com/2016-05-31-BioMarin-AnnouncesWithdrawal-of-Market-Authorization-Application-forKyndrisa-drisapersen-in-Europe Accessed July 12, 2017.

32. Press release drisapersen review FDA. http://investors .biomarin.com/2016-01-14-FDA-Issues-Complete-ResponseLetter-for-KyndrisaTM-for-Duchenne-Muscular-DystrophyAmenable-to-Exon-51-Skipping Accessed July 12, 2017.

33. FDA letter to Sarepta on eteplirsen approval. www.accessdata .fda.gov/drugsatfda_docs/appletter/2016/2064880rig1s000ltr .pdf Accessed July 12, 2017.

34. Sazani P, KP Ness, DL Weller, D Poage, K Nelson and AS Shrewsbury. (2011). Chemical and mechanistic toxicology evaluation of exon skipping phosphorodiamidate morpholino oligomers in mdx mice. Int J Toxicol 30:322333.
35. Crooke ST, BF Baker, TJ Kwoh, W Cheng, DJ Schulz, S Xia, N Salgado, HH Bui, CE Hart, et al. (2016). Integrated safety assessment of 2'-O-methoxyethyl chimeric antisense oligonucleotides in nonhuman primates and healthy human volunteers. Mol Ther 24:1771-1782.

36. Mojcik CF, MF Gourley, DM Klinman, AM Krieg, F Gmelig-Meyling and AD Steinberg. (1993). Administration of a phosphorothioate oligonucleotide antisense to murine endogenous retroviral MCF env causes immune effects in vivo in a sequence-specific manner. Clin Immunol Immunopathol 67:130-136.

37. Scientific advise and protocol assistance at EMA. www .ema.europa.eu/ema/index.jsp?curl=pages/regulation/general/ general_content_000049.jsp\&mid=WC0b01 ac05800229b9 Accessed July 12, 2017.

38. Orphan drug legislation EU. http://eur-lex.europa.eu/ LexUriServ/LexUriServ.do?uri=OJ:L:2000:018:0001:0005: en:PDF Accessed July 12, 2017.

39. Legislation. www.ema.europa.eu/docs/en_GB/document library/Report/2012/07/WC500130376.pdf Accessed July 12, 2017.

40. Fletcher S, MI Bellgard, L Price, AP Akkari and SD Wilton. (2017). Translational development of splice-modifying antisense oligomers. Expert Opin Biol Ther 17:15-30.

41. Aartsma-Rus A, A Ferlini, N Goemans, AM Pasmooij, DJ Wells, K Bushby, E Vroom and P Balabanov. (2014). Translational and regulatory challenges for exon skipping therapies. Hum Gene Ther 25:885-892.

42. EU legislation on medicine approval. https://ec.europa.eu/ health/sites/health/files/files/eudralex/vol-1/dir_2001_83_ consol_2012/dir_2001_83_cons_2012_en.pdf Accessed July $1 \overline{2}, 2017$.

43. Aartsma-Rus A, A Ferlini and E Vroom. (2014). Biomarkers and surrogate endpoints in Duchenne: meeting report. Neuromuscul Disord 24:743-745.

44. Ferlini A, KM Flanigan, H Lochmuller, F Muntoni, PA 't Hoen and E McNally. (2015). 204th ENMC International Workshop on Biomarkers in Duchenne Muscular Dystrophy 24-26 January 2014, Naarden, The Netherlands. Neuromuscul Disord 25:184-198.

45. Wu B, C Cloer, P Lu, S Milazi, M Shaban, SN Shah, L Marston-Poe, HM Moulton and QL Lu. (2014). Exon skipping restores dystrophin expression, but fails to prevent disease progression in later stage dystrophic dko mice. Gene Ther 21:785-793.

46. EMA DMD guidance. www.ema.europa.eu/docs/en_GB/ document_library/Scientific_guideline/2015/12/WC500199239 .pdf Accessed July 12, 2017.

47. Biomarker qualification process. http://www.ema.europa.eu/ docs/en_GB/document_library/Regulatory_and_procedural_ guideline/2009/10/WC500004201.pdf Accessed July 12, 2017.

48. Elsasser A, J Regnstrom, T Vetter, F Koenig, RJ Hemmings, M Greco, M Papaluca-Amati and M Posch. (2014). Adaptive clinical trial designs for European marketing authorization: a survey of scientific advice letters from the European Medicines Agency. Trials 15:383.

49. Hofer MP, C Jakobsson, N Zafiropoulos, S Vamvakas, T Vetter, J Regnstrom and RJ Hemmings. (2015). Regulatory watch: impact of scientific advice from the European Medicines Agency. Nat Rev Drug Discov 14:302-303.

50. Innovation Task Force. www.ema.europa.eu/ema/index .jsp?curl=pages/regulation/general/general_content_000334 .jsp\&mid=WC0b01ac05800ba1d9 Accessed July 12, 2017. 
51. PRIME. www.ema.europa.eu/ema/index.jsp?curl=pages/ regulation/general/general_content_000660.jsp\&mid= WC0b01ac05809f8439 Accessed July 12, 2017.

52. Orphan drug designation (EMA). www.ema.europa.eu/ema/ index.jsp?curl=pages/regulation/general/general_content_ 000029.jsp\&mid=WC0b01ac0580b18a41 Accessed July 12, 2017.

53. Mariz S, S Tsigkos and L Fregonese. (2014). The orphan framework as a new opportunity: an expert opinion. Expert Opin Orphan Drugs 2:1181-1186.

54. EMA SME tools. www.ema.europa.eu/ema/index.jsp?curl= pages/regulation/general/general_content_000059.jsp\&mid= WC0b01ac05800240cc Accessed July 12, 2017.

55. Jacobson RD and EL Feldman. (2016). Antisense oligonucleotides for Duchenne muscular dystrophy: why no neurologist should skip this. JAMA Neurol 73:259260.
Address correspondence to:

Pavel Balabanov, MD, PhD

Central Nervous System and Ophthalmology Scientific and Regulatory Management Department Human Medicines Evaluation Division European Medicines Agency 30 Churchill Place Canary Wharf London E14 5EU United Kingdom

E-mail: pavel.balabanov@ema.europa.eu

Received for publication June 21, 2017; accepted after revision July 12, 2017. 